\title{
Production and Hedging under Smooth Ambiguity Preferences
}

\author{
KIT PONG WONG *
}

This paper examines the optimal production and hedging decisions of the competitive firm facing ambiguous price and background risk. Ambiguity is modeled by a secondorder probability distribution that captures the firm's uncertainty about which of the subjective beliefs govern the price and background risk. Ambiguity preferences are modeled by the (second-order) expectation of a concave transformation of the (first-order) expected utility of profit conditional on each plausible subjective joint distribution of the price and background risk. When the background risk is additive in nature, we show that the separation theorem holds in that the firm's optimal production decision depends neither on the firm's attitude towards ambiguity nor on the incident of the underlying ambiguity. We derive necessary and sufficient conditions under which the firm's optimal forward position is completely characterized. When the background risk is multiplicative in nature, we derive sufficient conditions under which the firm reduces its optimal output level and opts for an under-hedge. Contrary to the conventional wisdom, we show that the behavior of the firm is affected by the introduction of ambiguity even when the firm is ambiguity neutral.

\section{INTRODUCTION}

Since the seminal work of Sandmo (1971), the behavior of the competitive firm has been the subject of considerable research in decision making under uncertainty. One important strand of this literature studies how the introduction of forward hedging affects the firm's

\footnotetext{
${ }^{*}$ Kit Pong Wong is a Professor of Finance and the Director of the School of Economics and Finance at the University of Hong Kong, Hong Kong. The author thanks Bob Webb (the editor) and an anonymous referee for their helpful comments and suggestions. The usual disclaimer applies.
}

JEL Classification: D21, D81, G13

Correspondence author, School of Economics and Finance, University of Hong Kong, Pokfulam Road, Hong Kong. Tel: +852-2859-1044, Fax: +852-2548-1152, e-mail: kpwong@econ.hku.hk 
production and hedging decisions (Danthine, 1978; Feder et al., 1980; Holthausen, 1979), from which two notable results emanate. First, the separation theorem states that the firm's optimal output level depends neither on the firm's preferences nor on the price distribution. Second, the full-hedging theorem asserts that the firm should completely eliminate its exposure to the price risk by adopting a full-hedge if the forward price is unbiased. ${ }^{1}$

While most of the extant models in the literature are developed within the expected utility paradigm, there are legitimate reasons to extend the analysis to the case wherein the firm is unable to unambiguously assign a probability distribution that uniquely describes the price uncertainty. To this end, we define uncertainty in the sense of Knight (1921) to be made up of two components, risk and ambiguity. ${ }^{2}$ Risk aversion is the aversion to a set of outcomes with a known probability distribution. Ambiguity aversion is the additional aversion to being unsure about the probabilities of outcomes. Dated back to the Ellsberg's (1961) paradox, ambiguity has been alluded to the violation of the independence axiom, which is responsible for the decision criterion being linear in the outcome probabilities. ${ }^{3}$ The distinction between the known-unknown and the unknown-unknown is relevant since individuals appear to prefer gambles with known rather than unknown probabilities. Indeed, ample experiments (Chow and Sarin, 2001; Einhorn and Hogarth, 1986; Sarin and Weber, 1993) and surveys (Chesson and Viscusi, 2003; Viscusi and Chesson, 1999) have documented convincing evidence that ambiguity aversion prevails.

\footnotetext{
${ }^{1}$ The full-hedging theorem is analogous to a well-known result in the insurance literature that a risk-averse individual fully insures at an actuarially fair price (Mossin, 1968).

${ }^{2}$ Knight (1921) points out that ambiguity is fundamentally different from risk. Specifically, risk relates to objective uncertainty, where outcome probabilities are known or can be estimated with confidence. In contrast, ambiguity relates to subjective uncertainty, where outcome probabilities are unknown, and decision makers are not sure which estimated models are correct.

${ }^{3}$ A vivid description of the Ellsberg's (1961) paradox is from Keynes (1921). Consider the following experiment with two urns, K and U. Urn K contains 50 red balls and 50 blue balls. Urn U contains 100 balls (all balls are either red or blue), but the exact numbers of red and blue balls are not disclosed. Subjects are asked to select from which urn they would like to draw a ball. They are rewarded if the color of their choice is drawn. In this experiment, subjects typically select urn K, revealing aversion to ambiguity. To see this, suppose that subjects believe that the probability of drawing a blue ball from urn $\mathrm{U}$ is $p$. Subjects should prefer to draw a red ball or a blue ball from urn $\mathrm{U}$ than from urn $\mathrm{K}$, depending on whether $p$ is smaller or greater than $1 / 2$, respectively. If $p=1 / 2$, subjects should be indifferent between the two urns. Since subjects choose to draw from urn K, such paradoxical behavior can only be rationalized by allowing multiple priors to be held by subjects on urn U. See also Dillenberger and Segal (2015) and Machina (2014).
} 
Klibanoff et al. (2005) have recently developed a powerful decision criterion known as "smooth ambiguity aversion" that is compatible with ambiguity averse preferences under uncertainty (hereafter referred to as the KMM model). The KMM model features the recursive structure that is far more tractable in comparison to other models of ambiguity such as the pioneering maxmin expected utility (or multiple-prior) model of Gilboa and Schmeidler (1989). ${ }^{4}$ Specifically, the KMM model represents ambiguity by a second-order probability distribution that captures a decision maker's uncertainty about which of the subjective beliefs govern the underlying risk. The KMM model then measures the decision maker's expected utility under ambiguity by taking the (second-order) expectation of a concave transformation of the (first-order) expected utility conditional on each plausible subjective distribution of the underlying risk. This recursive structure creates a crisp separation between ambiguity and ambiguity aversion, i.e., between beliefs and tastes, which allows these two attributes to be studied independently. Another nice feature of the KMM model is that we can apply the conventional techniques in the decision making under uncertainty in the context of ambiguity (Alary et al., 2013; Cherbonnier and Gollier, 2015; Gollier, 2011, 2014; Iwaki and Osaki, 2014; Snow, 2010, 2011; Taboga, 2005; Treich, 2010; Wong, 2015).

In this paper, we incorporate the KMM model into the competitive firm of Sandmo (1971) by assuming that the firm possesses smooth ambiguity preferences. Besides the price risk, the firm faces other sources of uncertainty that are aggregated into a single random variable. We refer to this as background risk, which can be either additive or multiplicative in nature (Wong, 2014). Examples of additive background risk abound. Some of the initial wealth of the competitive firm may be held in risky assets and thus creates an additive source of uncertainty (Chavas, 1985). The fixed cost of the competitive firm may also be random because the firm's physical assets can be ruined by natural disasters or fire (Wong, 1996). Examples of multiplicative background risk include revenue risk (Adam-Müller, 1997;

\footnotetext{
${ }^{4}$ Skiadas (2013) shows that smooth ambiguity preferences can be approximated by preferences admitting an expected utility representation in continuous-time or high-frequency models under Brownian or Poisson uncertainty.
} 
Wong, 2003), credit risk (Wong, 1997), and inflation risk (Adam-Müller, 2000; Battermann and Broll, 2001), all of which are shocks to the firm's cash flow in a multiplicative manner.

The firm faces uncertainty in the sense of Knight (1921) about the price and background risk. While it is reasonable to assume that the firm regards the price and background risk to be independent of each other for given subjective beliefs, they are deemed to be second-order dependent when the subjective beliefs vary. We show that this second-order dependence structure of the ambiguous price and background risk plays a pivotal role in shaping the firm's production and hedging decisions.

When the background risk is additive in nature, we show that the separation theorem holds in that the firm's optimal output level depends neither on the firm's attitude towards ambiguity nor on the incident of the underlying ambiguity. The full-hedging theorem does not hold in general. ${ }^{5}$ We derive necessary and sufficient conditions under which the firm's optimal forward position is completely characterized. When the background risk is multiplicative in nature, we derive sufficient conditions under which the firm reduces its optimal output level and opts for an under-hedge. Contrary to the conventional wisdom, we show that the behavior of the firm is affected by the introduction of ambiguity even when the firm is ambiguity neutral. This novel finding is driven by the second-order dependence structure between the ambiguous price and background risk, which is absent in the extant literature that focuses on a single source of ambiguity.

In a closely related paper, Osaki et al. (2015) examine the competitive firm's hedging demand for options under smooth ambiguity preferences when there is background risk that is additive and ambiguous. They show that the firm optimally uses no options should its exposure to the additive background risk be linearly related to that to the price risk under ambiguity. In the general case that these exposures are non-linearly related, they show that the firm includes options in its optimal hedge position to create the right curvature

\footnotetext{
${ }^{5}$ Wong (2015) shows that the full-hedging theorem holds if the ambiguity-averse firm faces only the ambiguous price risk. Osaki et al. (2015) show that the full-hedging theorem holds if the firm is risk neutral and the background risk is unambiguous.
} 
that better copes with the non-linearity arising from the second-order dependence structure between the ambiguous price and background risk. Like Osaki et al. (2015), we show in the case of additive ambiguous background risk that the separation theorem holds, and that the full-hedging theorem holds if the firm is risk neutral and the expected background risk is preserved as subjective beliefs vary. Unlike Osaki et al. (2015), we extend the analysis to the case of multiplicative ambiguous background risk, and focus on how forward hedging affects the firm's optimal production decision. Furthermore, we show that the ambiguityneutral firm may behave differently with and without ambiguity under multiple sources of ambiguity, which warrant a closer scrutiny in future research.

The rest of this paper is organized as follows. Section 2 delineates the KMM model of the competitive firm under ambiguous price and background risk. Section 3 characterizes the firm's optimal production and hedging decisions when the background risk is additive in nature. Section 4 examines the firm's optimal production and hedging decisions when the background risk is multiplicative in nature. The final section concludes.

\section{THE MODEL}

Consider the competitive firm of Sandmo (1971) within the context of the KMM model. There is one period with two dates, 0 and 1 . To begin, the firm produces a single commodity according to a deterministic cost function, $C(Q)$, where $Q \geq 0$ is the output level and $C(Q)$ is compounded to date 1 . The cost function, $C(Q)$, has the properties that $C(0)=C^{\prime}(0)=$ 0 , and $C^{\prime}(Q)>0$ and $C^{\prime \prime}(Q)>0$ for all $Q>0 .{ }^{6}$ At date 1 , the firm sells its entire output, $Q$, at the then prevailing spot price, $\tilde{P}$, per unit of output. ${ }^{7}$ The price risk, $\tilde{P}$, is distributed according to an objective cumulative distribution function (CDF), $F^{\circ}(P)$, over support $[\underline{P}, \bar{P}]$, where $0<\underline{P}<\bar{P}$. Let $\mathrm{E}_{F^{\circ}}(\cdot)$ be the expectation operator with respect to

\footnotetext{
${ }^{6}$ The strict convexity of the cost function is driven by the firm's production technology that exhibits decreasing returns to scale.

${ }^{7}$ Throughout the paper, random variables have a tilde $(\sim)$ while their realizations do not.
} 
$F^{\circ}(P)$

Besides the price risk, $\tilde{P}$, the firm faces other sources of risk that are aggregated into a single random variable, $\tilde{Z}$. We refer to $\tilde{Z}$ as the background risk that is independent of $\tilde{P}$. We allow $\tilde{Z}$ to be either additive or multiplicative in nature. Let $G^{\circ}(Z)$ be the objective CDF of $\tilde{Z}$ over support $[\underline{Z}, \bar{Z}]$ and $\mathrm{E}_{G^{\circ}}(\cdot)$ be the expectation operator with respect to $G^{\circ}(Z)$, where $\underline{Z}<0<\bar{Z}$. Throughout the paper, we assume that $\mathrm{E}_{G^{\circ}}(\tilde{Z})=0$.

While the background risk, $\tilde{Z}$, is neither hedgeable nor insurable, the firm can hedge against the price risk, $\tilde{P}$, by selling (purchasing if negative) $X$ units of its output forward at a predetermined forward price, $P^{f}$, per unit at date 0 , where $P^{f}=\mathrm{E}_{F^{\circ}}(\tilde{P})$ so that the forward price is unbiased. The firm's profit at date 1 is given by

$$
\Pi(\tilde{P}, \tilde{Z})=(1+\gamma \tilde{Z}) \tilde{P} Q-C(Q)+\left(P^{f}-\tilde{P}\right) X+(1-\gamma) \tilde{Z}
$$

where $\gamma=0$ or 1 , depending on whether the background risk is additive or multiplicative, respectively. ${ }^{8}$ We refer to $X$ as the firm's forward position, which is said to be an underhedge, a full-hedge, or an over-hedge, depending on whether $X$ is smaller than, equal to, or greater than the firm's output level, $Q$, respectively. The firm possesses a von NeumannMorgenstern utility function, $u(\Pi)$, defined over its profit at date 1 , $\Pi$, with $u^{\prime}(\Pi)>0$ and $u^{\prime \prime}(\Pi)<0$, indicating the presence of risk aversion.

The firm faces ambiguity in that it is uncertain about the objective CDFs, $F^{\circ}(P)$ and $G^{\circ}(Z)$. Specifically, let $F(P \mid \theta)$ and $G(Z \mid \theta)$ be the firm's subjective CDFs of $\tilde{P}$ and $\tilde{Z}$, respectively, where $\theta$ is the realization of an unknown parameter, $\tilde{\theta}$. The KMM model represents ambiguity by a second-order subjective $\operatorname{CDF}$ of $\tilde{\theta}, H(\theta)$, over support $[\underline{\theta}, \bar{\theta}]$ with $\underline{\theta}<\bar{\theta}$, which captures the firm's uncertainty about which of the subjective CDFs govern $\tilde{P}$ and $\tilde{Z}$. Following Gollier (2011) and Snow (2010, 2011), we assume that the firm's ambiguous beliefs are unbiased in the following sense:

$$
\int_{\underline{\theta}}^{\bar{\theta}} F(P \mid \theta) \mathrm{d} H(\theta)=F^{\circ}(P),
$$

\footnotetext{
${ }^{8}$ When $\gamma=1$, we assume that $1+\underline{Z}>0$.
} 
for all $P \in[\underline{P}, \bar{P}]$, and

$$
\int_{\underline{\theta}}^{\bar{\theta}} G(Z \mid \theta) \mathrm{d} H(\theta)=G^{\circ}(Z),
$$

for all $Z \in[\underline{Z}, \bar{Z}]$. Let $\mathrm{E}_{F}(\cdot \mid \theta), \mathrm{E}_{G}(\cdot \mid \theta)$, and $\mathrm{E}_{H}(\cdot)$, be the expectation operators with respect to the subjective CDFs, $F(P \mid \theta), G(Z \mid \theta)$, and $H(\theta)$, respectively.

While the price risk, $\tilde{P}$, and the background risk, $\tilde{Z}$, are first-order independent given a fixed value of the parameter, $\theta$, they become second-order dependent as $\theta$ varies. To see this, we calculate the covariance between $\tilde{P}$ and $\tilde{Z}$ :

$$
\begin{aligned}
& \int_{\underline{\theta}}^{\bar{\theta}} \int_{\underline{P}}^{\bar{P}} \int_{\underline{Z}}^{\bar{Z}}\left[P-\mathrm{E}_{F^{\circ}}(\tilde{P})\right]\left[Z-\mathrm{E}_{G^{\circ}}(\tilde{Z})\right] \mathrm{d} F(P \mid \theta) \mathrm{d} G(Z \mid \theta) \mathrm{d} H(\theta) \\
& =\int_{\underline{\theta}}^{\bar{\theta}} \int_{\underline{P}}^{\bar{P}} \int_{\underline{Z}}^{\bar{Z}}\left[P-\mathrm{E}_{F}(\tilde{P} \mid \theta)\right]\left[Z-\mathrm{E}_{G}(\tilde{Z} \mid \theta)\right] \mathrm{d} F(P \mid \theta) \mathrm{d} G(Z \mid \theta) \mathrm{d} H(\theta) \\
& \quad+\int_{\underline{\theta}}^{\bar{\theta}}\left[\mathrm{E}_{F}(\tilde{P} \mid \theta)-\mathrm{E}_{F^{\circ}}(\tilde{P})\right]\left[\mathrm{E}_{G}(\tilde{Z} \mid \theta)-\mathrm{E}_{G^{\circ}}(\tilde{Z})\right] \mathrm{d} H(\theta) \\
& =\operatorname{Cov}_{H}\left[\mathrm{E}_{F}(\tilde{P} \mid \tilde{\theta}), \mathrm{E}_{G}(\tilde{Z} \mid \tilde{\theta})\right],
\end{aligned}
$$

where $\operatorname{Cov}_{H}(\cdot, \cdot)$ is the covariance operator with respect to $H(\theta)$, and we have used Equations (2) and (3). It follows from Equation (4) that $\tilde{P}$ and $\tilde{Z}$ are second-order positively (negatively) dependent if $\operatorname{Cov}_{H}\left[\mathrm{E}_{F}(\tilde{P} \mid \tilde{\theta}), \mathrm{E}_{G}(\tilde{Z} \mid \tilde{\theta})\right]>(<) 0$. For example, this is the case when changes in $\theta$ affect $\mathrm{E}_{F}(\tilde{P} \mid \theta)$ and $\mathrm{E}_{G}(\tilde{Z} \mid \theta)$ in the same direction (opposite directions) for all $\theta \in[\underline{\theta}, \bar{\theta}]$.

The recursive structure of the KMM model implies that we can compute the firm's expected utility under ambiguity in three steps. First, we calculate the firm's expected utility for each pair of subjective CDFs of $\tilde{P}$ and $\tilde{Z}$ :

$$
U(\theta)=\int_{\underline{P}}^{\bar{P}} \int_{\underline{Z}}^{\bar{Z}} u[\Pi(P, Z)] \mathrm{d} F(P \mid \theta) \mathrm{d} G(Z \mid \theta),
$$


where $\Pi(P, Z)$ is given by Equation (1). Second, we transform each (first-order) expected utility obtained in Equation (5) by an ambiguity function, $\varphi(U)$, where $U$ is the firm's utility level. Finally, we take the (second-order) expectation of the transformed expected utility obtained in the second step with respect to the second-order subjective CDF of $\tilde{\theta}$. We can therefore state the firm's ex-ante decision problem as follows:

$$
\max _{Q \geq 0, X} \int_{\underline{\theta}}^{\bar{\theta}} \varphi[U(\theta)] \mathrm{d} H(\theta)
$$

where $U(\theta)$ is given by Equation (5). Inspection of the objective function of program (6) reveals that the effect of ambiguity, represented by $H(\theta)$, and that of ambiguity preferences, represented by $\varphi(U)$, can be separated and studied independently.

We say that the firm is ambiguity averse if, for any given output level, $Q$, the objective function of program (6) decreases when the firm's ambiguous beliefs, specified by $H(\theta)$, change in a way that induces a mean-preserving spread in the distribution of the firm's expected utility. According to this definition, Klibanoff et al. (2005) show that ambigu-

ity aversion implies that the ambiguity function, $\varphi(U)$, is concave in $U{ }^{9}$ The ambiguity function, $\varphi(U)$, satisfies that $\varphi^{\prime}(U)>0$ and $\varphi^{\prime \prime}(U) \leq 0$. The firm is ambiguity neutral or ambiguity averse, depending on whether $\varphi(U)=U$ or $\varphi^{\prime \prime}(U)<0$, respectively.

\section{ADDITIVE BACKGROUND RISK}

In this section, we examine the case wherein the background risk is additive in nature so that $\gamma=0$. The first-order conditions for program (6) with $\gamma=0$ are given by

$$
\int_{\underline{\theta}}^{\bar{\theta}} \int_{\underline{P}}^{\bar{P}} \int_{\underline{Z}}^{\bar{Z}} \varphi^{\prime}\left[U^{*}(\theta)\right] u^{\prime}\left[\Pi^{*}(P, Z)\right]\left[P-C^{\prime}\left(Q^{*}\right)\right] \mathrm{d} F(P \mid \theta) \mathrm{d} G(Z \mid \theta) \mathrm{d} H(\theta)=0,
$$

\footnotetext{
${ }^{9}$ When $\varphi(U)=-\eta^{-1} \exp (-\eta U)$, Klibanoff et al. (2005) show that the maxmin expected utility model of Gilboa and Schmeidler (1989) is the limiting case as the coefficient of absolute ambiguity aversion, $\eta$, approaches infinity under some conditions.
} 
and

$$
\int_{\underline{\theta}}^{\bar{\theta}} \int_{\underline{P}}^{\bar{P}} \int_{\underline{Z}}^{\bar{Z}} \varphi^{\prime}\left[U^{*}(\theta)\right] u^{\prime}\left[\Pi^{*}(P, Z)\right]\left[\mathrm{E}_{F^{\circ}}(\tilde{P})-P\right] \mathrm{d} F(P \mid \theta) \mathrm{d} G(Z \mid \theta) \mathrm{d} H(\theta)=0,
$$

where an asterisk $\left(^{*}\right)$ signifies an optimal level. The second-order conditions for program (6) are satisfied given the assumed properties of $\varphi(U), u(\Pi)$, and $C(Q)$.

We first examine the firm's optimal production decision. To this end, we add Equation (7) to Equation (8) to yield

$$
\left[\mathrm{E}_{F^{\circ}}(\tilde{P})-C^{\prime}\left(Q^{*}\right)\right] \int_{\underline{\theta}}^{\bar{\theta}} \int_{\underline{P}}^{\bar{P}} \int_{\underline{Z}}^{\bar{Z}} \varphi^{\prime}\left[U^{*}(\theta)\right] u^{\prime}\left[\Pi^{*}(P, Z)\right] \mathrm{d} F(P \mid \theta) \mathrm{d} G(Z \mid \theta) \mathrm{d} H(\theta)=0 .
$$

Since $\varphi^{\prime}(U)>0$ and $u^{\prime}(\Pi)>0$, Equation (9) implies that $C^{\prime}\left(Q^{*}\right)=\mathrm{E}_{F^{\circ}}(\tilde{P})$. We as such establish our first proposition.

Proposition 1. When the ambiguity-averse competitive firm can hedge the price risk by trading its output forward at the unbiased forward price, $\mathrm{E}_{F^{\circ}}(\tilde{P})$, introducing the additive background risk to the firm has no effect on its optimal output level, $Q^{*}$, which solves $C^{\prime}\left(Q^{*}\right)=\mathrm{E}_{F \circ}(\tilde{P})$.

The intuition for Proposition 1 is as follows. Since the firm can always sell the last unit of its output forward at the unbiased forward price, $\mathrm{E}_{F^{\circ}}(\tilde{P})$, the usual optimality condition applies in that the marginal cost of production, $C^{\prime}\left(Q^{*}\right)$, must be equated to the known marginal revenue, $\mathrm{E}_{F^{\circ}}(\tilde{P})$, which determines the optimal output level, $Q^{*}$. An immediate implication of Proposition 1 is that the firm's optimal production decision depends neither on the firm's attitude towards ambiguity nor on the incident to the underlying ambiguity. Proposition 1 as such extends the separation theorem of Danthine (1978), Feder et al. (1980), and Holthausen (1979) to the case of smooth ambiguity preferences and in the presence of ambiguous price and background risk (see also Osaki et al., 2015).

We now examine the firm's hedging decision. To this end, we differentiate the objective 
function of program (6) with respect to $X$, and evaluate the resulting derivative at $Q=Q^{*}$ and $X=Q^{*}$ to yield

$$
\begin{aligned}
& \left.\frac{\partial}{\partial X} \int_{\underline{\theta}}^{\bar{\theta}} \varphi[U(\theta)] \mathrm{d} H(\theta)\right|_{Q=Q^{*}, X=Q^{*}} \\
& =\int_{\underline{\theta}}^{\bar{\theta}} \varphi^{\prime}\left\{\mathrm{E}_{G}\left[u\left(\Pi^{*}+\tilde{Z}\right) \mid \theta\right]\right\} \mathrm{E}_{G}\left[u^{\prime}\left(\Pi^{*}+\tilde{Z}\right) \mid \theta\right]\left[\mathrm{E}_{F^{\circ}}(\tilde{P})-\mathrm{E}_{F}(\tilde{P} \mid \theta)\right] \mathrm{d} H(\theta) \\
& =-\operatorname{Cov}_{H}\left\{\varphi^{\prime}\left\{\mathrm{E}_{G}\left[u\left(\Pi^{*}+\tilde{Z}\right) \mid \tilde{\theta}\right]\right\} \mathrm{E}_{G}\left[u^{\prime}\left(\Pi^{*}+\tilde{Z}\right) \mid \tilde{\theta}\right], \mathrm{E}_{F}(\tilde{P} \mid \tilde{\theta})\right\}
\end{aligned}
$$

where $\Pi^{*}=\mathrm{E}_{F^{\circ}}(\tilde{P}) Q^{*}-C\left(Q^{*}\right)$, and the second equality follows from Equation $(2)$ and the property of the covariance operator. ${ }^{10}$ It follows from Equation (8) and the second-order conditions for program $(6)$ that $X^{*}>(<) Q^{*}$ if, and only if, the right-hand side of Equation (10) is positive (negative), thereby invoking the following proposition.

Proposition 2. When the ambiguity-averse competitive firm can hedge the price risk by trading its output forward at the unbiased forward price, $\mathrm{E}_{F^{\circ}}(\tilde{P})$, the firm's optimal forward position, $X^{*}$, is an over-hedge (under-hedge), i.e., $X^{*}>(<) Q^{*}$, if, and only if, the following condition holds:

$$
\operatorname{Cov}_{H}\left\{\varphi^{\prime}\left\{\mathrm{E}_{G}\left[u\left(\Pi^{*}+\tilde{Z}\right) \mid \tilde{\theta}\right]\right\} \mathrm{E}_{G}\left[u^{\prime}\left(\Pi^{*}+\tilde{Z}\right) \mid \tilde{\theta}\right], \mathrm{E}_{F}(\tilde{P} \mid \tilde{\theta})\right\}<(>) 0
$$

To see the intuition for Proposition 2, we define the following function:

$$
\Phi(\theta)=\int_{\underline{\theta}}^{\theta} \frac{\varphi^{\prime}\left\{\mathrm{E}_{G}\left[u\left(\Pi^{*}+\tilde{Z}\right) \mid x\right]\right\} \mathrm{E}_{G}\left[u^{\prime}\left(\Pi^{*}+\tilde{Z}\right) \mid x\right]}{\mathrm{E}_{H}\left\{\varphi^{\prime}\left\{\mathrm{E}_{G}\left[u\left(\Pi^{*}+\tilde{Z}\right) \mid \tilde{\theta}\right]\right\} \mathrm{E}_{G}\left[u^{\prime}\left(\Pi^{*}+\tilde{Z}\right) \mid \tilde{\theta}\right]\right\}} \mathrm{d} H(x),
$$

for all $\theta \in[\underline{\theta}, \bar{\theta}]$. It is evident from Equation (12) that $\Phi(\underline{\theta})=0, \Phi(\bar{\theta})=1$, and $\Phi^{\prime}(\theta)>0$ for all $\theta \in[\underline{\theta}, \bar{\theta}]$ so that we can interpret $\Phi(\theta)$ as a distorted second-order CDF of $\tilde{\theta}$. From Equations (8), (10), and (12), we have $X^{*}>(<) Q^{*}$ if, and only if,

$$
\mathrm{E}_{F \circ}(\tilde{P})>(<) \int_{\underline{\theta}}^{\bar{\theta}} \mathrm{E}_{F}(\tilde{P} \mid \theta) \mathrm{d} \Phi(\theta)
$$

\footnotetext{
${ }^{10}$ For any two random variables, $\tilde{X}$ and $\tilde{Y}$, we have $\operatorname{Cov}_{H}(\tilde{X}, \tilde{Y})=\mathrm{E}_{H}(\tilde{X} \tilde{Y})-\mathrm{E}_{H}(\tilde{X}) \mathrm{E}_{H}(\tilde{Y})$.
} 
where the right-hand side of condition (13) can be interpreted as the certainty equivalent spot price when the firm opts for a full-hedge, i.e., $X=Q^{*}$, taking the firm's ambiguity preferences and the underlying ambiguity into account. In the absence of the background risk, i.e., $\tilde{Z} \equiv 0$, Eq. (12) implies that $\Phi(\theta)=H(\theta)$ for all $\theta \in[\underline{\theta}, \bar{\theta}]$ so that there is no distortion. The unbiased forward price as such is equal to the certainty equivalent spot price under a full-hedge, thereby making $X^{*}=Q^{*}$ optimal. In the presence of the background risk, there is distortion that makes $\Phi(\theta)$ differ from $H(\theta)$. Using Equation (12), we have

$$
\begin{aligned}
\int_{\underline{\theta}}^{\bar{\theta}} \mathrm{E}_{F}(\tilde{P} \mid \theta) \mathrm{d} \Phi(\theta)= & \mathrm{E}_{F^{\circ}}(\tilde{P}) \\
& +\frac{\operatorname{Cov}_{H}\left\{\varphi^{\prime}\left\{\mathrm{E}_{G}\left[u\left(\Pi^{*}+\tilde{Z}\right) \mid \tilde{\theta}\right]\right\} \mathrm{E}_{G}\left[u^{\prime}\left(\Pi^{*}+\tilde{Z}\right) \mid \tilde{\theta}\right], \mathrm{E}_{F}(\tilde{P} \mid \tilde{\theta})\right\}}{\mathrm{E}_{H}\left\{\varphi^{\prime}\left\{\mathrm{E}_{G}\left[u\left(\Pi^{*}+\tilde{Z}\right) \mid \tilde{\theta}\right]\right\} \mathrm{E}_{G}\left[u^{\prime}\left(\Pi^{*}+\tilde{Z}\right) \mid \tilde{\theta}\right]\right\}} .
\end{aligned}
$$

We can interpret the second term on the left-hand side of Equation (14) as the risk premium demanded by the firm to compensate for its exposure to the ambiguous price and background risk. Condition (11) ensures that this term is negative (positive) so that the certainty equivalent spot price under a full-hedge, i.e., $X=Q^{*}$, is below (above) the unbiased forward price, thereby inducing the firm to optimally opt for an over-hedge (under-hedge), i.e., $X^{*}>(<) Q^{*}$.

Osaki et al. (2015) show that the full-hedging theorem of Danthine (1978), Feder et al. (1980), and Holthausen (1979) extends to the case of smooth ambiguity preferences and in the presence of ambiguous price and background risk if the firm is risk neutral, i.e., $u(\Pi)=\Pi$, and the expected background risk, $\mathrm{E}_{G}(\tilde{Z} \mid \theta)$, is preserved as $\theta$ varies. In this case, condition (11) becomes $\operatorname{Cov}_{H}\left\{\varphi^{\prime}\left[\Pi^{*}+\mathrm{E}_{G}(\tilde{Z} \mid \tilde{\theta})\right], \mathrm{E}_{F}(\tilde{P} \mid \tilde{\theta})\right\}=0$ so that $X^{*}=Q^{*}$. Indeed, condition (11) completely characterizes the firm's optimal forward position, thereby generalizing the findings of Osaki et al. (2015).

Before leaving this section, one novel implication is in order. Suppose that there is no ambiguity, i.e., the firm knows the objective $\operatorname{CDFs}, F^{\circ}(P)$ and $G^{\circ}(Z)$. In this benchmark 
case, the covariance term in condition (11) vanishes so that a full-hedge, i.e., $X^{*}=Q^{*}$, is optimal (see also Briys et al., 1993). We now resume the original case wherein ambiguity prevails. Suppose that the firm is ambiguity neutral in that $\varphi(U)=U$ and has a quadratic utility function, $u(\Pi)=a \Pi-b \Pi^{2} / 2$, where $a$ and $b$ are positive constants such that $u^{\prime}(\Pi)=$ $a-b \Pi>0$ for all relevant values of $\Pi$. From Proposition 1, the firm's optimal output level, $Q^{*}$, solves $C^{\prime}\left(Q^{*}\right)=\mathrm{E}_{F^{\circ}}(\tilde{P})$. Solving Equation (8) with $\varphi^{\prime}(U)=1$ and $u^{\prime}(\Pi)=a-b \Pi$ yields the firm's optimal forward position:

$$
X^{*}=Q^{*}+\frac{\operatorname{Cov}_{H}\left[\mathrm{E}_{F}(\tilde{P} \mid \tilde{\theta}), \mathrm{E}_{G}(\tilde{Z} \mid \tilde{\theta})\right]}{\operatorname{Var}_{F^{\circ}}(\tilde{P})},
$$

where $\operatorname{Var}_{F^{\circ}}(\cdot)$ is the variance operator with respect to $F^{\circ}(P)$. It follows from Equation (15) that $X^{*}>(<) Q^{*}$ if, and only if, $\operatorname{Cov}_{H}\left[\mathrm{E}_{F}(\tilde{P} \mid \tilde{\theta}), \mathrm{E}_{G}(\tilde{Z} \mid \tilde{\theta})\right]>(<) 0$, which is consistent with condition (11). As such, the ambiguity-neutral firm optimally opts for an over-hedge (under-hedge), not a full-hedge, when $\tilde{P}$ and $\tilde{Z}$ are second-order positively (negatively) dependent. In the presence of the ambiguous price and background risk, the behavior of the firm is affected by the introduction of ambiguity even under ambiguity neutrality. This is in stark contrast to the findings in the extant literature that focuses on a single source of ambiguity.

\section{MULTIPLICATIVE BACKGROUND RISK}

In this section, we examine the case wherein the background risk is multiplicative in nature so that $\gamma=1$. The first-order conditions for program (6) with $\gamma=1$ are given by

$$
\int_{\underline{\theta}}^{\bar{\theta}} \int_{\underline{P}}^{\bar{P}} \int_{\underline{Z}}^{\bar{Z}} \varphi^{\prime}\left[U^{*}(\theta)\right] u^{\prime}\left[\Pi^{*}(P, Z)\right]\left[(1+Z) P-C^{\prime}\left(Q^{*}\right)\right] \mathrm{d} F(P \mid \theta) \mathrm{d} G(Z \mid \theta) \mathrm{d} H(\theta)=0,
$$

and

$$
\int_{\underline{\theta}}^{\bar{\theta}} \int_{\underline{P}}^{\bar{P}} \int_{\underline{Z}}^{\bar{Z}} \varphi^{\prime}\left[U^{*}(\theta)\right] u^{\prime}\left[\Pi^{*}(P, Z)\right]\left[\mathrm{E}_{F^{\circ}}(\tilde{P})-P\right] \mathrm{d} F(P \mid \theta) \mathrm{d} G(Z \mid \theta) \mathrm{d} H(\theta)=0,
$$


where an asterisk $\left(^{*}\right)$ signifies an optimal level. The second-order conditions for program (6) are satisfied given the assumed properties of $\varphi(U), u(\Pi)$, and $C(Q)$.

We first examine the firm's optimal production decision. To this end, we add Equation (16) to Equation (17) to yield

$$
\begin{aligned}
& {\left[\mathrm{E}_{F^{\circ}}(\tilde{P})-C^{\prime}\left(Q^{*}\right)\right] \int_{\underline{\theta}}^{\bar{\theta}} \int_{\underline{P}}^{\bar{P}} \int_{\underline{Z}}^{\bar{Z}} \varphi^{\prime}\left[U^{*}(\theta)\right] u^{\prime}\left[\Pi^{*}(P, Z)\right] \mathrm{d} F(P \mid \theta) \mathrm{d} G(Z \mid \theta) \mathrm{d} H(\theta) } \\
= & -\int_{\underline{\theta}}^{\bar{\theta}} \int_{\underline{P}}^{\bar{P}} \int_{\underline{Z}}^{\bar{Z}} \varphi^{\prime}\left[U^{*}(\theta)\right] u^{\prime}\left[\Pi^{*}(P, Z)\right] P Z \mathrm{~d} F(P \mid \theta) \mathrm{d} G(Z \mid \theta) \mathrm{d} H(\theta) \\
= & -\mathrm{E}_{H}\left\{\varphi^{\prime}\left[U^{*}(\tilde{\theta})\right] \operatorname{Cov}_{F G}\left\{u^{\prime}\left[\Pi^{*}(\tilde{P}, \tilde{Z})\right] \tilde{P}, \tilde{Z} \mid \tilde{\theta}\right\}\right\} \\
& -\operatorname{Cov}_{H}\left\{\varphi^{\prime}\left[U^{*}(\tilde{\theta})\right] \mathrm{E}_{F G}\left\{u^{\prime}\left[\Pi^{*}(\tilde{P}, \tilde{Z})\right] \tilde{P} \mid \tilde{\theta}\right\}, \mathrm{E}_{G}(\tilde{Z} \mid \tilde{\theta})\right\},
\end{aligned}
$$

where $\mathrm{E}_{F G}(\cdot \mid \theta)$ and $\operatorname{Cov}_{F G}(\cdot, \cdot \mid \theta)$ are the expectation and covariance operators with respect to the joint CDF, $F(P \mid \theta) G(Z \mid \theta)$, respectively, and the second equality follows from Equation (3) and the fact that $\mathrm{E}_{G^{\circ}}(\tilde{Z})=0$. Since $\partial u^{\prime}\left[\Pi^{*}(P, Z)\right] P / \partial Z=u^{\prime \prime}\left[\Pi^{*}(P, Z)\right] P^{2} Q^{*}<0$, we have $\operatorname{Cov}_{F G}\left\{u^{\prime}\left[\Pi^{*}(\tilde{P}, \tilde{Z})\right] \tilde{P}, \tilde{Z} \mid \theta\right\}<0$ for all $\theta \in[\underline{\theta}, \bar{\theta}]$. Hence, the first term on the righthand side of Equation (18) is positive. The second term on the right-hand side of Equation (18) vanishes if $\mathrm{E}_{G}(\tilde{Z} \mid \theta)$ is preserved as $\theta$ varies, i.e., $\mathrm{E}_{G}(\tilde{Z} \mid \theta)=0$ for all $\theta \in[\underline{\theta}, \bar{\theta}]$. We as such establish the following proposition.

Proposition 3. When the ambiguity-averse competitive firm can hedge the price risk by trading its output forward at the unbiased forward price, $\mathrm{E}_{F^{\circ}}(\tilde{P})$, introducing the multiplicative background risk to the firm reduces its optimal output level, $Q^{*}$, such that $C^{\prime}\left(Q^{*}\right)<\mathrm{E}_{F^{\circ}}(\tilde{P})$, if the expected background risk, $\mathrm{E}_{G}(\tilde{Z} \mid \theta)$, is preserved as the parameter, $\theta$, varies.

To see the intuition for Proposition 3, we define the following two functions:

$$
F^{u}(P, Z \mid \theta)=\int_{\underline{P}}^{P} \int_{\underline{Z}}^{Z} \frac{u^{\prime}\left[\Pi^{*}(x, y)\right]}{\mathrm{E}_{F G}\left\{u^{\prime}\left[\Pi^{*}(\tilde{P}, \tilde{Z})\right] \mid \theta\right\}} \mathrm{d} F(x \mid \theta) \mathrm{d} G(y \mid \theta),
$$


for all $(P, Z) \in[\underline{P}, \bar{P}] \times[\underline{Z}, \bar{Z}]$ and $\theta \in[\underline{\theta}, \bar{\theta}]$, and

$$
\Psi(\theta)=\int_{\underline{\theta}}^{\theta} \frac{\varphi^{\prime}\left[U^{*}(x)\right] \mathrm{E}_{F G}\left\{u^{\prime}\left[\Pi^{*}(\tilde{P}, \tilde{Z})\right] \mid x\right\}}{\mathrm{E}_{H}\left\{\varphi^{\prime}\left[U^{*}(\tilde{\theta})\right] \mathrm{E}_{F G}\left\{u^{\prime}\left[\Pi^{*}(\tilde{P}, \tilde{Z})\right] \mid \tilde{\theta}\right\}\right\}} \mathrm{d} H(x)
$$

for all $\theta \in[\underline{\theta}, \bar{\theta}]$. It is evident from Equations (19) and (20) that we can interpret $F^{u}(P, Z \mid \theta)$ as a distorted joint $\mathrm{CDF}$ of $\tilde{P}$ and $\tilde{Z}$, and $\Psi(\theta)$ as a distorted CDF of $\tilde{\theta}$. Substituting Equations (19) and (20) into Equation (18) yields

$$
\mathrm{E}_{F^{\circ}}(\tilde{P})+\int_{\underline{\theta}}^{\bar{\theta}} \mathrm{E}_{F^{u}}(\tilde{P} \tilde{Z} \mid \theta) \mathrm{d} \Psi(\theta)=C^{\prime}\left(Q^{*}\right)
$$

where $\mathrm{E}_{F^{u}}(\cdot \mid \theta)$ is the expectation operator with respect to $F^{u}(P, Z \mid \theta)$. The left-hand side of Equation (21) can be interpreted as the certainty equivalent marginal revenue, taking the firm's ambiguity preferences and the underlying ambiguity into account. Equation (21) is the usual optimality condition such that the firm's optimal output level, $Q^{*}$, equates the marginal cost of production, $C^{\prime}\left(Q^{*}\right)$, to the certainty equivalent marginal revenue. Even though the firm can always sell the last unit of its output forward at the unbiased forward price, $\mathrm{E}_{F^{\circ}}(\tilde{P})$, the firm's marginal revenue remains stochastic because of the multiplicative background risk. The second term on the left-hand side of Equation (21) is the risk premium demanded by the firm to compensate for its exposure to the ambiguous price and background risk. Using Equations (18) and (21), we have

$$
\begin{aligned}
\int_{\underline{\theta}}^{\bar{\theta}} \mathrm{E}_{F^{u}}(\tilde{P} \tilde{Z} \mid \theta) \mathrm{d} \Psi(\theta)= & \frac{\mathrm{E}_{H}\left\{\varphi^{\prime}\left[U^{*}(\tilde{\theta})\right] \operatorname{Cov}_{F G}\left\{u^{\prime}\left[\Pi^{*}(\tilde{P}, \tilde{Z})\right] \tilde{P}, \tilde{Z} \mid \tilde{\theta}\right\}\right\}}{\mathrm{E}_{H}\left\{\varphi^{\prime}\left[U^{*}(\tilde{\theta})\right] \mathrm{E}_{F G}\left\{u^{\prime}\left[\Pi^{*}(\tilde{P}, \tilde{Z})\right] \mid \tilde{\theta}\right\}\right\}} \\
& +\frac{\operatorname{Cov}_{H}\left\{\varphi^{\prime}\left[U^{*}(\tilde{\theta})\right] \mathrm{E}_{F G}\left\{u^{\prime}\left[\Pi^{*}(\tilde{P}, \tilde{Z})\right] \tilde{P} \mid \tilde{\theta}\right\}, \mathrm{E}_{G}(\tilde{Z} \mid \tilde{\theta})\right\}}{\mathrm{E}_{H}\left\{\varphi^{\prime}\left[U^{*}(\tilde{\theta})\right] \mathrm{E}_{F G}\left\{u^{\prime}\left[\Pi^{*}(\tilde{P}, \tilde{Z})\right] \mid \tilde{\theta}\right\}\right\}} .
\end{aligned}
$$

The first term on the right-hand side of Equation (22) is the component of the risk premium that is driven mainly by risk aversion, which is always negative in the presence of the multiplicative background risk. The second term is the component of the risk premium 
that is driven mainly by the ambiguous background risk. It goes away when the background risk is unambiguous in the sense that $\mathrm{E}_{G}(\tilde{Z} \mid \theta)=0$ for all $\theta \in[\underline{\theta}, \bar{\theta}]$. In this case, the risk premium in Equation $(21)$ is negative so that $C^{\prime}\left(Q^{*}\right)<\mathrm{E}_{F^{\circ}}(\tilde{P})$.

We now examine the firm's hedging decision. To this end, we differentiate the objective function of program (6) with respect to $X$, and evaluate the resulting derivative at $Q=Q^{*}$ and $X=Q^{*}$ to yield

$$
\begin{aligned}
& \left.\frac{\partial}{\partial X} \int_{\underline{\theta}}^{\bar{\theta}} \varphi[U(\theta)] \mathrm{d} H(\theta)\right|_{Q=Q^{*}, X=Q^{*}} \\
& =\int_{\underline{\theta}}^{\bar{\theta}} \int_{\underline{P}}^{\bar{P}} \int_{\underline{Z}}^{\bar{Z}} \varphi^{\prime}\left\{\mathrm{E}_{F G}\left[u\left(\Pi^{*}+\tilde{P} \tilde{Z} Q^{*}\right) \mid \theta\right]\right\} \\
& \quad \times u^{\prime}\left(\Pi^{*}+P Z Q^{*}\right)\left[\mathrm{E}_{F^{\circ}}(\tilde{P})-P\right] \mathrm{d} F(P \mid \theta) \mathrm{d} G(Z \mid \theta) \mathrm{d} H(\theta) \\
& =-\mathrm{E}_{H}\left\{\varphi^{\prime}\left\{\mathrm{E}_{F G}\left[u\left(\Pi^{*}+\tilde{P} \tilde{Z} Q^{*}\right) \mid \tilde{\theta}\right]\right\} \operatorname{Cov}_{F G}\left[u^{\prime}\left(\Pi^{*}+\tilde{P} \tilde{Z} Q^{*}\right), \tilde{P} \mid \tilde{\theta}\right]\right\} \\
& -\operatorname{Cov}_{H}\left\{\varphi^{\prime}\left\{\mathrm{E}_{F G}\left[u\left(\Pi^{*}+\tilde{P} \tilde{Z} Q^{*}\right) \mid \tilde{\theta}\right]\right\} \mathrm{E}_{F G}\left[u^{\prime}\left(\Pi^{*}+\tilde{P} \tilde{Z} Q^{*}\right) \mid \tilde{\theta}\right], \mathrm{E}_{F}(\tilde{P} \mid \tilde{\theta})\right\},
\end{aligned}
$$

where $\Pi^{*}=\mathrm{E}_{F^{\circ}}(\tilde{P}) Q^{*}-C\left(Q^{*}\right)$. In the following proposition, we derive sufficient conditions under which the right-hand side of Equation (23) is negative.

Proposition 4. When the ambiguity-averse competitive firm can hedge the price risk by trading its output forward at the unbiased forward price, $\mathrm{E}_{F^{\circ}}(\tilde{P})$, the firm's optimal forward position, $X^{*}$, is an under-hedge, i.e., $X^{*}<Q^{*}$, if both the expected price risk and background risk, $\mathrm{E}_{F}(\tilde{P} \mid \theta)$ and $\mathrm{E}_{G}(\tilde{Z} \mid \theta)$, are preserved as the parameter, $\theta$, varies, and the utility function, $u(\Pi)$, exhibits prudence, i.e., $u^{\prime \prime \prime}(\Pi)>0$.

Proof. Since $\mathrm{E}_{G}(\tilde{Z} \mid \theta)=0$ for all $\theta \in[\underline{\theta}, \bar{\theta}]$ and $u^{\prime \prime \prime}(\Pi)>0$, we have $\partial \mathrm{E}_{G}\left[u^{\prime}\left(\Pi^{*}+\right.\right.$ $\left.\left.P \tilde{Z} Q^{*}\right) \mid \theta\right] / \partial P=\operatorname{Cov}_{G}\left[u^{\prime \prime}\left(\Pi^{*}+P \tilde{Z} Q^{*}\right), \tilde{Z} \mid \theta\right] Q^{*}>0$ for all $\theta \in[\underline{\theta}, \bar{\theta}]$. Hence, the first term on the right-hand side of Equation $(23)$ is negative. Since $\mathrm{E}_{F}(\tilde{P} \mid \theta)$ is preserved as $\theta$ 
varies, i.e., $\mathrm{E}_{F}(\tilde{P} \mid \theta)=\mathrm{E}_{F^{\circ}}(\tilde{P})$ for all $\theta \in[\underline{\theta}, \bar{\theta}]$, the second term on the right-hand side of Equation (23) vanishes. It then follows from Equations (17) and (23) and the second-order conditions for program (6) that $X^{*}<Q^{*}$.

To show that the second-order dependence structure between the ambiguous price and background risk renders the behavior of the firm to be affected by the introduction of ambiguity even under ambiguity neutrality, suppose that the firm is ambiguity neutral in that $\varphi(U)=U$, and has a quadratic utility function, $u(\Pi)=a \Pi-b \Pi^{2} / 2$, where $a$ and $b$ are positive constants such that $u^{\prime}(\Pi)=a-b \Pi>0$ for all relevant values of $\Pi$. When there is no ambiguity, the firm knows the objective CDFs, $F^{\circ}(P)$ and $G^{\circ}(Z)$. In this benchmark case, we substitute $\varphi^{\prime}(U)=1$ and $u^{\prime}(\Pi)=a-b \Pi$ into Equation (16) to yield

$$
\left[\mathrm{E}_{F^{\circ}}(\tilde{P})-C^{\prime}\left(Q^{\circ}\right)\right]\left(a-b \Pi^{\circ}\right)-b Q^{\circ} \mathrm{E}_{F^{\circ}}\left(\tilde{P}^{2}\right) \mathrm{E}_{G^{\circ}}\left(\tilde{Z}^{2}\right)=0
$$

where $\Pi^{\circ}=\mathrm{E}_{F^{\circ}}(\tilde{P}) Q^{\circ}-C\left(Q^{\circ}\right)$, and $Q^{\circ}$ is the firm's optimal output level. It is evident from Equation (24) that $C^{\prime}\left(Q^{\circ}\right)<\mathrm{E}_{F^{\circ}}(\tilde{P})$. Eq. (17) implies that the firm's optimal forward position is a full-hedge, i.e., $X^{\circ}=Q^{\circ}$.

When ambiguity prevails, we substitute $\varphi^{\prime}(U)=1$ and $u^{\prime}(\Pi)=a-b \Pi$ into Equations (16) and (17) to yield

$$
\begin{aligned}
& {\left[\mathrm{E}_{F^{\circ}}(\tilde{P})-C^{\prime}\left(Q^{*}\right)\right]\left(a-b \Pi^{*}\right)-b Q^{*} \mathrm{E}_{H}\left[\mathrm{E}_{F}\left(\tilde{P}^{2} \mid \tilde{\theta}\right) \mathrm{E}_{G}\left(\tilde{Z}^{2} \mid \tilde{\theta}\right)\right]} \\
& +\left\{a-b \Pi^{*}+b\left[C^{\prime}\left(Q^{*}\right) Q^{*}-C\left(Q^{*}\right)\right]\right\} \operatorname{Cov}_{H}\left[\mathrm{E}_{F}(\tilde{P} \mid \tilde{\theta}), \mathrm{E}_{G}(\tilde{Z} \mid \tilde{\theta})\right] \\
& +b\left(\frac{Q^{*}-X^{*}}{Q^{*}}\right)^{2} Q^{*} \operatorname{Var}_{F}(\tilde{P})=0,
\end{aligned}
$$

and

$$
X^{*}=Q^{*}\left\{1+\frac{\operatorname{Cov}_{H}\left[\mathrm{E}_{F}\left(\tilde{P}^{2} \mid \tilde{\theta}\right)-\mathrm{E}_{F}(\tilde{P} \mid \tilde{\theta}) \mathrm{E}_{F^{\circ}}(\tilde{P}), \mathrm{E}_{G}(\tilde{Z} \mid \tilde{\theta})\right]}{\operatorname{Var}_{F^{\circ}}(\tilde{P})}\right\}
$$


where $\Pi^{*}=\mathrm{E}_{F^{\circ}}(\tilde{P}) Q^{*}-C\left(Q^{*}\right)$. Equation (26) implies that a full-hedge, i.e., $X^{*}=Q^{*}$, is in general suboptimal. To see this, suppose that $\mathrm{E}_{F}(\tilde{P} \mid \theta)=\mathrm{E}_{F^{\circ}}(\tilde{P})$ for all $\theta \in[\underline{\theta}, \bar{\theta}]$. The covariance term on the right-hand side of Equation (26) becomes $\operatorname{Cov}_{H}\left[\operatorname{Var}_{F}(\tilde{P} \mid \tilde{\theta}), \mathrm{E}_{G}(\tilde{Z} \mid \tilde{\theta})\right]$. In this case, an over-hedge (under-hedge), i.e., $X^{*}>(<) Q^{*}$, is optimal if, and only if, $\operatorname{Cov}_{H}\left[\operatorname{Var}_{F}(\tilde{P} \mid \tilde{\theta}), \mathrm{E}_{G}(\tilde{Z} \mid \tilde{\theta})\right]>(<) 0$.

Eq. (25) implies that the firm's optimal output level, $Q^{*}$, is likely to be different from $Q^{\circ}$. To see this, suppose that $\mathrm{E}_{G}(\tilde{Z} \mid \theta)=0$ for all $\theta \in[\underline{\theta}, \bar{\theta}]$. In this case, Equation (26) reduces to $X^{*}=Q^{*}$ and Equation $(25)$ reduces to

$$
\begin{aligned}
& {\left[\mathrm{E}_{F^{\circ}}(\tilde{P})-C^{\prime}\left(Q^{*}\right)\right]\left(a-b \Pi^{*}\right)-b Q^{*} \mathrm{E}_{F^{\circ}}\left(\tilde{P}^{2}\right) \mathrm{E}_{G^{\circ}}\left(\tilde{Z}^{2}\right)} \\
& -b Q^{*} \operatorname{Cov}_{H}\left[\mathrm{E}_{F}\left(\tilde{P}^{2} \mid \tilde{\theta}\right), \mathrm{E}_{G}\left(\tilde{Z}^{2} \mid \tilde{\theta}\right)\right]=0 .
\end{aligned}
$$

From Equations (24) and (27) and the second-order conditions for program (6), we have $Q^{*}<(>) Q^{\circ}$ if, and only if, $\operatorname{Cov}_{H}\left[\mathrm{E}_{F}\left(\tilde{P}^{2} \mid \tilde{\theta}\right), \mathrm{E}_{G}\left(\tilde{Z}^{2} \mid \tilde{\theta}\right)\right]>(<) 0$.

\section{CONCLUSION}

In this paper, we examine the production and hedging decisions of the competitive firm under price and background risk when the firm can trade its output forward at the unbiased forward price. We allow the background risk to be either additive or multiplicative in nature. The firm possesses smooth ambiguity preferences à la Klibanoff et al. (2005). The KMM model represents ambiguity by a second-order probability distribution that captures the firm's uncertainty about which of the subjective beliefs govern the price and background risk. The KMM model then measures the firm's expected utility under ambiguity by taking the (second-order) expectation of a concave transformation of the (first-order) expected utility of profit conditional on each plausible subjective joint distribution of the price and background risk. 
When the background risk is additive in nature, we show that the separation theorem holds in that the firm's optimal production decision depends neither on the firm's attitude towards ambiguity nor on the incident of the underlying ambiguity. We derive necessary and sufficient conditions under which the firm's optimal forward position is completely characterized. When the background risk is multiplicative in nature, we derive sufficient conditions under which the firm reduces its optimal output level and opts for an underhedge. Contrary to the conventional wisdom, we show that the behavior of the firm is affected by the introduction of ambiguity even when the firm is ambiguity neutral. This novel finding is driven by the second-order dependence structure between the ambiguous price and background risk, which is absent in the extant literature that focuses on a single source of ambiguity.

\section{REFERENCES}

Adam-Müller, A. F. A. (1997). Export and hedging decisions under revenue and exchange rate risk: A note. European Economic Review, 41, 1421-1426.

Adam-Müller, A. F. A. (2000). Hedging price risk when real wealth matters. Journal of International Money and Finance, 19, 549-560.

Alary, D., Gollier, C., \& Treich, N. (2013). The effect of ambiguity aversion on insurance and self-protection. Economic Journal, 123, 1188-1202.

Battermann, H. L., \& Broll, U. (2001). Inflation risk, hedging, and exports. Review of Development Economics, 5, 355-362.

Briys, E., Crouhy, M., \& Schlesinger, H. (1993). Optimal hedging in a futures market with background noise and basis risk. European Economic Review, 37, 949-960.

Chavas, J.-P. (1985). On the theory of the competitive firm under uncertainty when initial wealth is random. Southern Economic Journal, 51, 818-827. 
Cherbonnier, F., \& Gollier, C. (2015). Decreasing aversion under ambiguity. Journal of Economic Theory, 157, 606-623.

Chesson, H. W., \& Viscusi, W. K. (2003). Commonalities in time and ambiguity aversion for long-term risks. Journal of Risk and Uncertainty, 24, 57-71.

Chow, C. C., \& Sarin, R. K. (2001). Comparative ignorance and the Ellsberg Paradox. Journal of Risk and Uncertainty, 22, 129-139.

Danthine, J.-P. (1978). Information, futures prices, and stabilizing speculation. Journal of Economic Theory, 17, 79-98.

Dillenberger, D., \& Segal, U. (2015). Recursive ambiguity and Machina's examples. International Economic Review, 56, 55-61.

Einhorn, H. J., \& Hogarth, R. M. (1986). Decision making under ambiguity. Journal of Business, 59, S225-S250.

Ellsberg, D. (1961). Risk, ambiguity, and the Savage axioms. Quarterly Journal of Economics, 75, 643-669.

Feder, G., Just, R. E., \& Schmitz, A. (1980). Futures markets and the theory of the firm under price uncertainty. Quarterly Journal of Economics, 94, 317-328.

Gilboa, I., \& Schmeidler, D. (1989). Maxmin expected utility with a non-unique prior. Journal of Mathematical Economics, 18, 141-153.

Gollier, C. (2011). Portfolio choices and asset prices: the comparative statics of ambiguity aversion. Review of Economic Studies, 78, 1329-1344.

Gollier, C. (2014). Optimal insurance design of ambiguous risks. Economic Theory, 57, $555-576$.

Holthausen, D. M. (1979). Hedging and the competitive firm under price uncertainty. American Economic Review, 69, 989-995.

Iwaki, H., \& Osaki, Y. (2014). The dual theory of the smooth ambiguity model. Economic 
Theory, 56, 275-289.

Keynes, J. M. (1921). A treatise on probability. London: Macmillan.

Klibanoff, P., Marinacci, M., \& Mukerji, S. (2005). A smooth model of decision making under ambiguity. Econometrica, 73, 1849-1892.

Knight, F. H. (1921). Risk, uncertainty and profit. Boston: Houghton Mifflin.

Machina, M. J. (2014). Ambiguity aversion with three or more outcomes. American Economic Review, 104, 3814-3840.

Mossin, J. (1968). Aspects of rational insurance purchasing. Journal of Political Economy, $76,553-568$.

Osaki, Y., Wong, K. P., \& Yi, L. (2015). Hedging and the competitive firm under ambiguous price and background risk. Working paper, University of Hong Kong.

Sandmo, A. (1971). On the theory of the competitive firm under price uncertainty. American Economic Review, 61, 65-73.

Sarin, R. K., \& Weber, M. (1993). Effects of ambiguity in market experiments. Management Science, 39, 602-615.

Skiadas, C. (2013). Smooth ambiguity aversion toward small risks and continuous-time recursive utility. Journal of Political Economy, 121, 775-792.

Snow, A. (2010). Ambiguity and the value of information. Journal of Risk and Uncertainty, 40, 133-145.

Snow, A. (2011). Ambiguity aversion and the propensities for self-insurance and selfprotection. Journal of Risk and Uncertainty, 42, 27-43.

Taboga, M. (2005). Portfolio selection with two-stage preferences. Finance Research Letters, $2,152-164$.

Treich, N. (2010). The value of a statistical life under ambiguity aversion. Journal of 
Environmental Economics and Management, 59, 15-26.

Viscusi, W. K., \& Chesson, H. W. (1999). Hopes and fears: the conflicting effects of risk ambiguity. Theory and Decision, 47, 153-178.

Wong, K. P. (1996). Background risk and the theory of the competitive firm under uncertainty. Bulletin of Economic Research, 48, 241-251.

Wong, K. P. (1997). On the determinants of bank interest margins under credit and interest rate risks. Journal of Banking and Finance, 21, 251-271.

Wong, K. P. (2003). Currency hedging with options and futures. European Economic Review, 47, 833-839.

Wong, K. P. (2014). Hedging and the competitive firm under correlated price and background risk. Decisions in Economics and Finance, 37, 329-340.

Wong, K. P. (2015). Ambiguity and the value of hedging. Journal of Futures Markets, 35, $839-848$. 